\title{
Drug Extravasation in a Large General Hospital in Hunan, China: A Retrospective Survey
}

\author{
Zhihong Gong (D) \\ Jinghui Zhang $\mathbb{D}^{1-3}$ \\ Jianmei Hou ${ }^{1,4}$ \\ Shujie Chen' \\ Zixin $\mathrm{Hu}$ (D) $^{\prime}$ \\ Xiaoya Kong' \\ Guiyuan $\mathrm{Ma}^{\prime}$ \\ Lingxia Luo ${ }^{1,5}$
}

'Teaching and Research Section of Clinical Nursing, Xiangya Hospital of Central South University, Changsha, Hunan, People's Republic of China;

${ }^{2}$ National Clinical Research Center for Geriatric Disorders, Xiangya Hospital of Central South University, Changsha, Hunan, People's Republic of China; ${ }^{3}$ Xiangya Nursing School, Central South University, Changsha, Hunan, People's Republic of China; ${ }^{4}$ Department of Thoracic Oncology, Xiangya Hospital of Central South University, Changsha, Hunan, People's Republic of China; ${ }^{5}$ Department of Emergency Medicine, Xiangya Hospital of Central South University, Changsha, Hunan, People's Republic of China
Correspondence: Jinghui Zhang Teaching and Research Section of Clinical Nursing, Xiangya Hospital of Central South University, 87 Xiangya St, Kaifu District, Changsha, 4I0008, Hunan,

People's Republic of China

Tel +86 I5874032339

Email zhangjh-1206@I63.com
Background and Aim: Drug extravasation is one of the most common complications of intravenous therapy, which can lead to severe tissue injury if inappropriately treated. This study analyzes the current situation of extravasation and the risk factors affecting the severity of extravasation to provide a theoretical basis for carrying out prospective research, reducing the severity of drug extravasation, and strengthening the management of drug extravasation. Materials and Methods: We retrieved the data on extravasation from January 2016 to December 2020 from the hospital's safe infusion management system. We used nonparametric tests to assess the differences in the severity of drug extravasation among each variable and performed a multivariate analysis using multivariate ordered logistic regression. Results: Extravasation occurred in $0.038 \%$ (263/694,043) of patients, including 203 cases of mild extravasation $(77.2 \%), 57$ cases of moderate extravasation $(21.7 \%)$, and 3 cases of severe extravasation $(1.1 \%)$. The main diseases of the patients with extravasation were cancer $(24.7 \%)$, neurological-related diseases $(19.4 \%)$, circulatory-related diseases $(14.8 \%)$, and digestive-related diseases $(14.1 \%)$; the main extravasated drugs were hypotonic or hypertonic drugs $(31.9 \%)$ and contrast media $(27.8 \%)$; the infusion tools of extravasation were indwelling needles $(92.0 \%)$ and steel needles $(8.0 \%)$. The multi-factor analysis showed that close to joints, patients' age $\leq 6$ or age $>65$, cancer, neurological-related diseases, circulatory-related diseases, antineoplastic agents, hypotonic or hypertonic drugs and strong acid or alkali drugs were independent risk factors for more severe extravasation. The nurses' age and first identified by nurse were nurse-related factors that influenced the severity of drug extravasation

Conclusion: To prevent the occurrence of drug extravasation and reduce its severity, the nurses should strengthen the learning of emergency plans related drug extravasation, strengthen inspections of high-risk patients. Besides, the managers should strengthen the risk warning management of high-risk extravasated drugs.

Keywords: extravasation, risk factors, retrospective cohort study, the severity level

\section{Introduction}

Drug extravasation is one of the common complications of intravenous fluid therapy. It refers to the leakage of medicines from the blood, causing damage to the surrounding tissues, outside the venous lumen. ${ }^{1,2}$ The main symptoms include pain, tingling or burning sensation, and edema around the intravenous injection site. If not properly treated, extravasation can lead to tissue necrosis. The tissue damage can be classified into minor, superficial, and reversible skin lesions. Severe injury may lead to large full-thickness skin ulcers and damage to adjacent structures such as tendons, muscles, joints, and peripheral nerves ${ }^{3,4}$ Besides, scar formation can lead to contractures and deformities. Moreover, severe injuries caused by 
extravasation often require surgical intervention, such as debridement, reconstruction, or even amputation, ${ }^{5}$ thus resulting in prolonged hospital stays ${ }^{6}$ and increased morbidity and costs. ${ }^{7}$ In the UK, about $65 \%$ of clinical negligence claims in pediatric surgery result in payment to the claimant, with $4 \%$ of claims being made due to extravasation events. ${ }^{8}$

The incidence of extravasation ranges from $0.01 \%$ to $6 \%{ }^{9}$ High rates $(11 \%)$ have been observed in young children receiving intravenous fluids. ${ }^{3,10}$ The physiological and biochemical principles of drug extravasation are mainly twofold: on the one hand, the needle tip punctures the blood vessel, resulting in the entry of the drug from the vessel into the perivascular tissue. On the other hand, it is related to the physicochemical properties of the drug, the cytotoxic effect of the drug, which causes damage to the vascular endothelium; the different $\mathrm{pH}$ or osmotic pressure of the drug imbalances the acid-base or osmotic pressure inside and outside the vascular endothelial cells, resulting in damage to the vascular endothelium or changes in vascular permeability, which leads to drug extravasation. ${ }^{1,9}$ Previous studies have found that parenteral nutrition is the main cause of extravasation damage in neonatal units, while chemotherapy is the main cause of extravasation damage in oncology and hematology units. $^{11}$

Fortunately, most extravasation damage can be prevented with appropriate placement techniques and prophylaxis. Moreover, prompt recognition and appropriate treatment can prevent further tissue damage, pain, and even limb loss. ${ }^{10}$ Studies have shown that the severity of drug extravasation depends on the types of the extravasated drug, the duration of extravasation, and the amount of exudation. ${ }^{6,12}$ It has been suggested that active interventions, such as elevation of the affected limb, hot and cold compresses, and local closure with drug antagonists during the "necrotic interval" 3 after drug extravasation can reduce tissue damage. ${ }^{11,12}$ To reduce the severity of tissue damage, drug extravasation risk factors must be quickly identified and aggressively treated.

This study retrospectively analyzed the drug extravasation in Xiangya Hospital, China, in the past five years aiming to explore the relevant factors affecting the severity of drug extravasation and to provide a theoretical basis for carrying out prospective research, reducing the severity of drug extravasation, and strengthening the management of drug extravasation.

\section{Materials and Methods}

\section{Study Population}

This retrospective analysis, which included 263 inpatients' medical records from Xiangya Hospital, was performed between January 2016 and December 2020. All medical records were screened in detail for parameters including age and disease of the patients, the types of the extravasated drugs, the injection sites, the professional title and the age of the nurses. All data come from the safe infusion management system. The nurses reporting drug extravasation to this system were the one infuse the drug or/and the ones care the patients during infusion. Inclusion criteria: 1) all drug extravasation occurred in our hospital; 2) drug extravasation occurred from January 2016 to December 2020. Exclusion criteria: 1) Records with incomplete information; 2) Patients who were lost to follow-up.

\section{Assessment of the Severity of Extravasation}

The severity of drug extravasation was classified into three categories: ${ }^{3,4}$ mild, characterized by little pain and swelling, but no erythema or blisters; moderate, characterized by local inflammation of an area $<10 \mathrm{~cm}$ in diameter, with moderate tenderness, swelling, but no necrosis; severe, characterized by severe pain and significant swelling, blistering and possible skin necrosis.

\section{Classification of Patients' Disease}

Referring to the International Classification of Diseases, 11th Revision (ICD-11), patients' diseases were classified into 12 categories according to their main disease at the time of hospitalization: cancer, nervous system, circulatory system, digestive system, respiratory system, genitourinary system, endocrine system, locomotor system, diseases of the five senses and appendages, skin and wounds, immune diseases, and other (maternal and prematurity-related).

\section{Type of Extravasated Drugs}

In the present study, extravasated drugs were divided into six categories. ${ }^{1,4,13}$ Contrast media were divided into a separate category based on practicality. Other drugs are classified as antineoplastic (eg, Cisplatin, Doxorubicin, etc.) and non-antineoplastic agents. Non-antineoplastic agents are further divided into four categories: 1) inotropic agents (eg, Norepinephrine, Dopamine, etc.); 2) Strong acid or alkali drugs: strong acid drugs refer to $\mathrm{pH}<5.0$ 
(eg, Vancomycin, etc.), strong alkali drugs refer to $\mathrm{pH}>9.0$ (eg, Sodium Bicarbonate, Ganciclovir, etc.); 3) hypotonic or hypertonic drugs: hypotonic drugs refer to osmolarity $<250 \mathrm{mOsm} / \mathrm{L}$ (eg, 0.45\% Sodium Chloride Solution, etc.), hypertonic drugs refer to osmolality $>600 \mathrm{mOsm} / \mathrm{L}$ (such as Parenteral Nutrition Solution, 20\% Mannitol, etc.); 4) other drugs (such as Ringer's Solution, etc.).

\section{Ethical Statement}

This study informed all participants of the purpose of the study and obtained the consent of the participants; The clinical data and basic information of all the participants are strictly confidential. This study was approved by the Medical Ethics Committee of Xiangya Hospital, Central South University, Changsha, China. All procedures were in compliance with the Declaration of Helsinki.

\section{Statistical Analyses}

All statistical analyses were performed using SPSS 22.0 (SPSS Inc., Chicago, IL). The measurement data were expressed as mean \pm standard deviation. The statistical descriptions of the enumeration data were expressed as frequency and percentage. The $t$-test was used to compare the means of continuous variables and normally distributed data; otherwise, the Mann-Whitney $U$-test or KruskalWallis test was used. Statistically significant variables (which had a two-tailed $\mathrm{P}<0.05$ ) and variables that have clinical significance based on the medical literature were retained in the final multivariate model. Multiple ordered logistic regression analysis with the Wald test method was used to estimate the unadjusted odds ratios (ORs) and 95\% confidence intervals (CIs) for the association between all potential risk factors and severity of extravasation.

\section{Results}

\section{General Population Data}

This study included 694,043 patients who received the infusion. A total of 263 cases of drug extravasation were identified. The incidence of drug extravasation was $0.038 \%$, including 203 cases of mild drug extravasation (77.2\%), 57 cases of moderate drug extravasation (21.7\%), and 3 cases of severe drug extravasation (1.1\%). All patients with extravasation had a good prognosis, and no limb disability. The three cases of severe drug extravasation were financially compensated.

Cancer including leukemia (19), lung cancer (17), breast cancer (8), cervical cancer (5), and other cancers (16).
Neurological-related diseases including cerebral infarction (17), craniocerebral trauma (11), intracerebral hemorrhage (11), meningitis (4), and others (8); Circulatory-related diseases including hypertension (15), coronary heart disease (8), after heart surgery (4), acute myocardial infarction (4), aortic dissection (2), others (6). Digestive-related disease including gastric ulcer (8), liver cirrhosis (7), viral hepatitis (6), gastrointestinal bleeding (4), others (12).

Antineoplastic agents including Epirubicin (4), Paclitaxel (3), Carboplatin (3), Oxaliplatin (3), Etoposide (2), Vinorelbine (2), Cyclophosphamide (2), Methotrexate (2), and others (4); Inotropic agents including Dopamine (9), Noradrenaline (7), Amiodarone (4), Adrenaline (3) and Pituitrin (2), Hypotonic or hypertonic drugs including Parenteral nutrition solution (35), 10\% potassium chloride (7), Calcium (7), 50\% Glucose (7), Iron sucrose (7), 20\% Mannitol (6), Converted Sugar Electrolytes (4), Albumin (3), Globulin (3), and others (5); Strong acid or alkali drugs including Sodium Bicarbonate (11), Ampicillin (8), Acyclovir (6), Vancomycin (3), Ganciclovir (1); All the contrast agents were Iohexol.

The average age of patients with extravasation was $49.81 \pm 23.22$ years. The patients aged $\leq 6$ years or $>65$ years accounted for $36.5 \%$. The patients with extravasation suffered from the four main categories of diseases: cancer $(24.7 \%)$, diseases of the nervous system $(19.4 \%)$, circulatory system (14.8\%), and digestive system (14.1\%). The main extravasated drugs were hypotonic or hypertonic drugs $(31.9 \%)$ and contrast agents $(27.8 \%)$. The infusion tools of the drug extravasation were peripheral venous indwelling needles $(92.0 \%)$ and steel needles $(8.0 \%)$. The mean age of the nurses of drug extravasation was $27.21 \pm$ 4.73 years (95\% CI 26.64 27.78) and a median age of 27. The details are shown in Table 1.

\section{Univariate Analysis}

The univariate analysis compared the severity of drug extravasation under different gender, different age groups, different diseases and other factors. $\mathrm{P}<0.05$ showed that the severity of drug extravasation was statistically different. The results showed that the severity of drug extravasation was related to patients' age, patients' diseases, earliest identified personnel, types of extravasated drugs, injection sites close to joints, nurses' professional title, and nurses' age (all $\mathrm{P}<0.05$, Table 1 ). In the present study, variables with $\mathrm{P}<0.05$ in the univariate analysis were retained in the multivariate ordered logistic regression model. 
Table I Characteristics of Extravasation and Univariate Analysis of the Severity of Extravasation in the Last 5 Years

\begin{tabular}{|c|c|c|c|c|c|c|c|}
\hline \multicolumn{2}{|l|}{ Variable } & \multirow{3}{*}{$\begin{array}{l}\mathbf{n} \\
147 \\
116\end{array}$} & \multirow{3}{*}{$\begin{array}{c}\text { Mild (\%) } \\
\text { II } 4 \text { (77.5\%) } \\
89(76.7 \%)\end{array}$} & \multirow{3}{*}{$\begin{array}{c}\text { Moderate (\%) } \\
31(21.1 \%) \\
26(22.4 \%)\end{array}$} & \multirow{3}{*}{$\begin{array}{c}\text { Severe (\%) } \\
2(1.4 \%) \\
\text { I (0.9\%) }\end{array}$} & \multirow{3}{*}{$\frac{\mathbf{Z} / \mathbf{H}}{-0.135^{\mathrm{a}}}$} & \multirow{3}{*}{$\frac{\text { P-value }}{0.893}$} \\
\hline Gender & Male & & & & & & \\
\hline & Female & & & & & & \\
\hline \multirow[t]{2}{*}{ Patients' age (years) } & Age $\leq 6$ or age $>65$ & 96 & $67(69.8 \%)$ & 27 (28.1\%) & $2(2.1 \%)$ & $-2.197^{\mathrm{a}}$ & 0.028 \\
\hline & Others & 167 & $136(81.4 \%)$ & $30(18.0 \%)$ & I $(0.6 \%)$ & & \\
\hline \multirow[t]{5}{*}{ Patients' diseases } & Cancer & 65 & 41 (63.1\%) & $22(33.8 \%)$ & $2(3.1 \%)$ & $14.222^{\mathrm{b}}$ & $<0.001$ \\
\hline & Neurological-related diseases & 51 & 39 (75.5\%) & II (2I.5\%) & I (2.0\%) & & \\
\hline & Circulatory-related diseases & 39 & 29 (74.4\%) & $10(25.6 \%)$ & 0 & & \\
\hline & Digestive-related disease & 37 & $31(83.8 \%)$ & $6(16.2 \%)$ & 0 & & \\
\hline & Other diseases & 71 & $63(88.7 \%)$ & 8 (II.3\%) & 0 & & \\
\hline \multirow{3}{*}{$\begin{array}{l}\text { Earliest identified } \\
\text { personnel }\end{array}$} & Patients & 44 & $29(65.9 \%)$ & $15(34.1 \%)$ & 0 & $10.167^{\mathrm{b}}$ & 0.006 \\
\hline & Nurses & 204 & $166(81.4 \%)$ & $36(17.6 \%)$ & $2(1.0 \%)$ & & \\
\hline & Others & 15 & $8(53.3 \%)$ & $6(40.0 \%)$ & I (6.7\%) & & \\
\hline \multirow{6}{*}{$\begin{array}{l}\text { Types of extravasated } \\
\text { drugs }\end{array}$} & Antineoplastic agents & 25 & 14 (56.0\%) & $10(40.0 \%)$ & I (4.0\%) & $17.608^{\mathrm{b}}$ & $<0.001$ \\
\hline & Inotropic agents: & 25 & 19 (76.0\%) & $6(24.0 \%)$ & 0 & & \\
\hline & Hypotonic or hypertonic drugs & 84 & $58(69.0 \%)$ & $25(29.8 \%)$ & I (I.2\%) & & \\
\hline & Strong acid or alkali drugs & 29 & $20(69.0 \%)$ & $8(27.6 \%)$ & I (3.4\%) & & \\
\hline & Contrast media & 73 & 67 (91.8\%) & $6(8.2 \%)$ & 0 & & \\
\hline & Other drugs & 27 & $25(92.6 \%)$ & $2(7.4 \%)$ & 0 & & \\
\hline \multirow[t]{2}{*}{ Infusion tools } & Steel needles & 21 & 14 (66.7\%) & 7 (33.3\%) & 0 & $-1.150^{\mathrm{a}}$ & 0.250 \\
\hline & $\begin{array}{l}\text { Peripheral venous Indwelling } \\
\text { needle }\end{array}$ & 242 & I89 (78.1\%) & $50(20.7 \%)$ & $3(1.2 \%)$ & & \\
\hline \multirow[t]{5}{*}{ Injection sites } & Left upper limb & 89 & $66(74.2 \%)$ & $21(23.6 \%)$ & $2(2.2 \%)$ & $5.187^{b}$ & 0.159 \\
\hline & Right upper limb & 144 & 117 (8I.2\%) & $26(18.1 \%)$ & I (0.7\%) & & \\
\hline & Lower left limb & 17 & $10(58.8 \%)$ & 7 (4I.2\%) & 0 & & \\
\hline & Lower right limb & 10 & 7 (70.0\%) & $3(30.0 \%)$ & 0 & & \\
\hline & Other parts & 3 & $3(100.0 \%)$ & 0 & 0 & & \\
\hline \multirow{2}{*}{$\begin{array}{l}\text { Injection sites close to } \\
\text { joints }\end{array}$} & Yes & 169 & $138(81.7 \%)$ & $31(18.3 \%)$ & 0 & $-2.417^{\mathrm{a}}$ & 0.016 \\
\hline & No & 94 & 65 (69.1\%) & $26(27.7 \%)$ & $3(3.2 \%)$ & & \\
\hline \multirow[t]{3}{*}{ Nurses' professional title } & Nurse & 87 & 59 (67.8\%) & $26(29.9 \%)$ & $2(2.3 \%)$ & $7.287^{b}$ & 0.026 \\
\hline & Senior nurse & 142 & II 8 (83.1\%) & $23(16.2 \%)$ & I $(0.7 \%)$ & & \\
\hline & Supervisor nurse & 34 & $26(76.5 \%)$ & 8 (23.5\%) & 0 & & \\
\hline \multirow{3}{*}{$\begin{array}{l}\text { Nurses' age } \\
\text { (years) }\end{array}$} & Age $\leq 25$ & 103 & $68(66.0 \%)$ & 32 (31.1\%) & $3(2.9 \%)$ & $12.504^{\mathrm{b}}$ & 0.002 \\
\hline & $26<$ age $\leq 35$ & $|4|$ & 119 (84.4\%) & $22(15.6 \%)$ & 0 & & \\
\hline & Age $>35$ & 19 & $16(84.2 \%)$ & $3(15.8 \%)$ & 0 & & \\
\hline
\end{tabular}

Notes: ${ }^{a} \mathrm{Z}$ value; ${ }^{\mathrm{b}} \mathrm{H}$ value.

Abbreviations: Z, test statistics of Mann-Whitney U-test; H, test statistics of Kruskal-Wallis test.

There were 67 elderly patients, 8 of whom had cognitive impairment. There was a statistically significant difference $(p<0.05)$ in the severity of drug extravasation between elderly patients with or without cognitive impairment. The details are shown in Table 2.

\section{Multivariate Logistic Regression Analysis}

Multivariate analysis was performed with the severity of drug extravasation as the response variable and the factors with significant univariate analysis results as explanatory variables. A parallel line test was $\chi^{2}=10.774, \mathrm{P}=0.768$, which satisfied the hypothesis of multiple ordered logistics regression parallel lines. The results of the multivariate analysis are shown in Table 3, close to joints ( $\mathrm{OR}=3.319,95 \%$ CI: $1.625 \sim 6.779)$, patients' age $\leq 6$ or age $>65(\mathrm{OR}=3.016,95 \%$ CI: 1.193 6.623), cancer ( $\mathrm{OR}=2.721,95 \%$ CI: $1.932 \sim 7.943)$, or neurological-related diseases $(\mathrm{OR}=3.455,95 \% \mathrm{CI}$ : 1.166 10.236), or circulatory-related diseases $(\mathrm{OR}=3.467$, 
Table 2 Mann-Whitney U-test of Whether the Elderly Have Cognitive Impairment and the Severity of Drug Extravasation

\begin{tabular}{|l|c|c|c|c|c|c|}
\hline Cognitive Impairment & $\mathbf{n}$ & Mild (\%) & Moderate (\%) & Severe (\%) & Z & P-value \\
\hline No & 59 & $46(78.0 \%)$ & $13(22.0 \%)$ & 0 & 11.124 & 0.004 \\
Yes & 8 & $3(37.5 \%)$ & $4(50.0 \%)$ & $1(12.5 \%)$ & & \\
\hline
\end{tabular}

Abbreviation: Z, test statistics of Mann-Whitney U-test.

Table 3 Multivariate Ordered Logistic Regression Analysis of Factors Influencing the Severity of Drug Extravasation

\begin{tabular}{|c|c|c|c|c|}
\hline Variable & B & Wald & $\mathbf{P}$ & OR $(95 \% \mathrm{CI})$ \\
\hline Injection sites close to joints (Reference: no) & 1.200 & 10.836 & 0.001 & $3.319(1.625 \sim 6.779)$ \\
\hline Nurses' professional title (Reference: supervisor nurse) & 0.342 & 0.788 & 0.375 & $\mathrm{I} .408(0.66 \mathrm{I} 2.997)$ \\
\hline Nurses' age (years) & -1.054 & 5.341 & 0.021 & $0.348(0.143 \sim 0.852)$ \\
\hline Patients' age (years) (Reference: others) & $\mathrm{I} .104$ & 6.104 & 0.013 & $3.016(1.193 \sim 6.623)$ \\
\hline \multicolumn{5}{|l|}{ Patients' diseases (Reference: others diseases) } \\
\hline Cancer & 1.001 & 3.354 & 0.047 & 2.721 ( $1.932 \sim 7.943)$ \\
\hline Neurological-related diseases & 1.240 & 5.003 & 0.025 & $3.455(1.166 \sim 10.236)$ \\
\hline Circulatory-related diseases & 1.243 & 4.201 & 0.040 & $3.467(1.056 \sim 11.383)$ \\
\hline Digestive-related disease & 0.630 & 0.901 & 0.342 & $1.878(0.5 \mathrm{I} \mid \mathrm{l}, 8.899)$ \\
\hline \multicolumn{5}{|l|}{ Earliest identified personnel (Reference: others) } \\
\hline Patients & -0.105 & 0.021 & 0.884 & $0.900(0.218 \sim 3.716)$ \\
\hline Nurses & -1.382 & 2.288 & 0.030 & $0.25 \mathrm{I}(0.102 \sim 0.542)$ \\
\hline \multicolumn{5}{|l|}{ Types of extravasated drugs (Reference: other drugs) } \\
\hline Antineoplastic agents & 2.369 & 7.880 & 0.005 & $10.692(2.044 \sim 55.913)$ \\
\hline Inotropic agents & 1.172 & 2.256 & 0.133 & $3.229(0.699 \sim 14.909)$ \\
\hline Hypotonic or hypertonic drugs & 1.533 & 4.879 & 0.027 & $4.633(1.189 \sim 18.062)$ \\
\hline Strong acid or alkali drugs & 1.232 & 0.164 & 0.039 & $3.428(1 . \mid 35 \sim 21.131)$ \\
\hline Contrast media & 0.176 & 0.048 & 0.826 & I. $192(0.249 \sim 5.715)$ \\
\hline
\end{tabular}

Notes: The independent variables in the multivariate ordered logistic regression model were coadded as the following: injection sites close to joints (no $=0$, yes $=\mathrm{I}$ ), nurses' professional titles (nurse $=\mathrm{I}$, senior nurse $=2$, supervisor nurse $=3$ ), patients' age (years) (age $\leq 6$ or age $>65=I$, others $=0$ ), patients' diseases (cancer $=I$, neurological-related diseases $=2$, circulatory-related diseases $=3$, digestive-related disease $=4$, others diseases $=5$ ), earliest identified personnel (patients=I, nurses $=2$, others $=3$ ), types of extravasated drugs (antineoplastic agents $=I$, inotropic agents $=2$, hypotonic or hypertonic drugs $=3$, strong acid or alkali drugs $=4$, contrast media $=5$, other drugs $=6$ ).

Abbreviations: $\mathrm{Cl}$, confidence interval; $\mathrm{OR}$, odd ratio.

95\% CI: 1.056 11.383), antineoplastic agents $(\mathrm{OR}=10.692$, 95\% CI: 2.044 55.913), hypotonic or hypertonic drugs (OR = 4.633, 95\% CI: $1.189 \sim 18.062$ ) or strong acid or alkali drugs (OR $=3.428,95 \%$ CI: 1.135 21.131) were independent risk factors increasing the severity of drug extravasation. Nurses' age $(\mathrm{OR}=0.348,95 \% \mathrm{CI}: 0.143 \sim 0.852)$, first identified by nurse $(\mathrm{OR}=0.251,95 \% \mathrm{CI}: 0.102 \sim 0.542)$ were independent protective factors in reducing the severity of drug extravasation.

\section{Discussion}

Drug extravasation is one of the common complications of clinical intravenous therapy. Although most of the extravasation events were mild drug extravasation in this study,
$21.7 \%$ of patients developed skin blisters and swollen and stiff limbs. Soft tissue injury occurred in $1.1 \%$ of patients. Although there are many prevention and management programs, no fully effective treatment has been developed. Therefore, management of risk factors for drug extravasation severity should be enhanced to reduce the severity of extravasation and alleviate patients' suffering.

\section{Enhance the Care of Patients at High Risk of Extravasation}

The results show that the patients' age $\leq 6$ years or $>65$ years is a risk factor for increasing the severity of drug extravasation. Consistent with the research results of Goutos et al, ${ }^{3}$ infants and young children have relatively thin blood vessels thus 
making it difficult to perform puncture and more likely to damage blood vessels. ${ }^{14}$ Moreover, infants and young children are more likely to move and have poor compliance; thus, it is difficult to properly fix the infusion site during infusion. On the other hand, elderly patients have degraded body functions, loose skin prone to needle displacement, hardened blood vessels and narrowed lumen, and reduced blood fluidity. Consequently, the risk of extravasation in older population is greatly increased. Moreover, the results show that cognitive impairment is an influencing factor affecting the severity of drug extravasation in elderly patients. Cognitive impairment decrease the compliance to the perfusion and delays the extravasation diagnosis. Therefore, extravasation could be more severe. Besides, these two groups of patients cannot accurately express pain and discomfort, so it is more difficult to detect extravasation at an early stage. ${ }^{4,14}$ Therefore, vascular access device should be carefully selected when performing intravenous therapy. Peripherally inserted central catheters, which can reduce the risk of drug extravasation, are recommended for infusion in elderly patients and low cooperative infants. Besides, the catheter should be properly fixed to prevent drug extravasation caused by catheter dislodgement. Also, it is necessary to strengthen inspection for the elderly and infants so that drug extravasation can be timely detected and treated, thus reducing the severity of drug extravasation and reduce patients' pain.

In the present study, the patients with cancer $(24.7 \%)$, neurological-related diseases (19.4\%), and circulatory-related diseases $(14.8 \%)$ were at high risk of developing extravasation. This was consistent with previous studies, which suggested that the patients with chronic diseases, such as liver disease or ischemic encephalopathy, heart disease and tumor diseases, etc., are at higher risk of extravasation. ${ }^{15}$ This study shows that the risk of severe drug extravasation in cancer patients was 2.721 times that of other common diseases. Cancer patients were prone to drug extravasation due to repeated infusion, blood sampling, and long-term infusion of irritating antineoplastic agents, which can damage the intima of venous vessels and make the veins more sclerotic, brittle, atrophied, and thin. ${ }^{9}$ We also found that the risk of severe drug extravasation in patients with neurological-related diseases and circulatory-related diseases was 3.455-3.467 times higher than that of other common diseases. These patients had a long course of the disease and often require infusions of medications, including many inotropic agents, hypotonic or hypertonic drugs and strong acid or alkali drugs, which increase the permeability of the blood vessel walls and risk of drug extravasation. ${ }^{1,3}$ Therefore, it is necessary to strengthen inspection, reasonably select vascular access equipment, select appropriate puncture site (thick and straight blood vessels) for patients with cancer or chronic diseases.

\section{Strengthen the Management of High Risk Extravasation Drugs}

The results of the study showed that low or high osmolarity drugs $(31.9 \%)$, antineoplastic agents $(9.5 \%)$, and strong acidic and alkaline drugs $(11.0 \%)$ were independent risk factors that aggravated the severity of extravasation. This is generally consistent with the findings of Yan et al, ${ }^{16}$ who suggested that the severity of extravasation is associated with a large number of drugs or specific drugs (high permeability, high risk, low $\mathrm{pH}$, etc.). Human blood is isotonic with an osmolarity of $285 \mathrm{mOsm} / \mathrm{L}$. Hypotonic fluids can easily flow into the vascular endothelium, resulting in swelling and bursting of the endothelium. When hypertonic fluid is administered, water from the endothelial cell layer moves into the bloodstream, diluting the hypertonic drug and causing contraction and dehydration of the endothelial cells, followed by phlebitis and subsequent extravasation of the drug, then causing sclerosis and pain in the bleeding limb. ${ }^{17}$ Studies have also shown that patients receiving drugs with a $\mathrm{pH}$ below 4.1 or above 9.0 are at higher risk of extravasation. ${ }^{17}$ Antineoplastic agents, which are mostly corrosive or strong irritants, have the most serious degree of tissue damage. This study showed that antineoplastic agents are 10.692 times more likely to aggravate the severity of drug extravasation than common drugs. Therefore, when infusing high-risk extravasated drugs, a central venous catheter should be used to protect the blood vessels and reduce the risk of drug extravasation. ${ }^{2}$ Managers should develop a safe infusion early warning system, which can assess the high risk factors of drug extravasation of patients, and then warning nurses to choose the central venous channel reasonably, so as to avoid drug extravasation. Besides, the managers should formulate emergency plans for drug extravasation. Once drug extravasation occurs, nurses can accurately and quickly deal with drug extravasation, prevent further tissue damage of extravasated drugs and reduce the severity of drug extravasation. For patients who refuse to choose the central venous channel for high-risk extravasation drugs, the patients should be informed of the risk and signed an informed consent form for the infusion of high-risk extravasation drugs.

Our data showed that contrast agents $(27.0 \%)$ and inotropic agents (12.5\%) were also common extravasated drugs, which is consistent with Hwang et $\mathrm{al}^{18}$ and Behzadi et al. ${ }^{19}$ Hwang et al ${ }^{18}$ indicated that the incidence of extravasation of 
CT contrast media was $0.23 \%$, and that female, age $>60$ years, and ICU ward were independent risk factors for contrast media extravasation. Behzadi et $\mathrm{al}^{19}$ showed that the rate of extravasation was nearly six times higher for iodine-based contrast agents $(0.26 \%)$ than for gadolinium-based contrast agents $(0.045 \%)$. Infusion of inotropic agents, such as dopamine, causes peripheral vasoconstriction and vasospasm, which in turn leads to tissue ischemia and hypoxia. This results in increased permeability of the tubular wall and then extravasation of the drug into the tissue interstitium, which can cause tissue damage or even necrosis in severe cases. ${ }^{20}$ Therefore, selecting the appropriate size and material of vascular access device and avoiding the joint site during puncture are essential for preventing extravasation of the contrast agent by high-pressure push injection. Studies have shown that pushing contrast through a high-pressure-resistant PICC catheter can effectively prevent contrast extravasation and enhance CT images' contrast effect. ${ }^{21}$ If choosing to use an indwelling needle to push the contrast medium, the recommended size is larger than $22 \mathrm{G}$ and the connector should be high-pressure resistant. ${ }^{2}$

\section{Improve Nurses' Ability to Prevent and Manage Drug Extravasation}

The present study showed that two factors, first identified by nurses and nurses' age, appeared to be more favorable in mitigating drug extravasation. Nurses can identify early drug extravasation more accurately than patients and their families, thus being able to timely recommend correct treatment measures to prevent further development of drug extravasation. In our hospital, older nurses tend to work longer. Therefore, they always have richer work experience and a higher level of knowledge about drug extravasation, which allows them to take more accurate and timely measures and reduce the severity of drug extravasation. Prevention, early diagnosis and appropriate treatment can significantly reduce the consequences of extravasation, so it is crucial to provide guidance and training to nurses and patients on recognition of symptoms, prevention and management of extravasation. ${ }^{12}$

The results of multivariate analysis showed that the infusion site close to the joint was an independent risk factor for the severity of drug extravasation. In the present study, $64.3 \%$ of drug extravasation sites were close to joint, which is consistent with the findings of Loubani et $\mathrm{al}^{22}$ who found that in the majority of drug extravasation events $(174 / 204,85.3 \%)$, the infusion site was located in the elbow or popliteal fossa. The movement of the joint often increases the risk of displacement or detachment of the infusion catheter, so it is more prone to drug extravasation. Therefore, it is recommended to avoid the joint when performing vein puncture and properly fix the catheter is very important.

The results showed that the main infusion tools used were indwelling needles $(92.0 \%)$ and steel needles $(8.0 \%)$ when extravasation of drugs occurred. Therefore, it is necessary to choose the appropriate infusion tools when infusing high-risk extravasated drugs. If the indwelling needle is needed, it is necessary to properly fix the catheter, strengthen the inspection and closely observe the infusion. However, it has also been shown that extravasation of the PORT (implantable venous access port) occurs due to misplacement of the non-injurious needle. ${ }^{23}$ Therefore, it is important to strengthen the training of nurses in PORT maintenance skills and make sure to draw back blood before infusion.

\section{Limitations and Prospects}

First of all, this study is a single-center study, and there may be selection bias, which underestimates or overestimates the clinical outcome. In addition, there may be a lack of information (such as the minimum duration of extravasation, the patient's hospitalization course and compliance, etc.) and the non-standard nature of the collected data during the retrospective study, which may affect the results of this study. However, the multivariate ordered logistic regression model of this study is meaningful, so the results of the study still have clinical value.

In the future, we will carry out prospective case-control studies on the basis of this study, and analyze the risk factors of the severity of drug extravasation more rigorously and comprehensively. The results of this study also help us improve the safe infusion management system and help us better manage drug extravasation.

\section{Conclusions}

Patients with cancer, neurological-related diseases, and circulatory-related diseases are at high risk of more severe drug extravasation damage, while those receiving low or high osmolarity drugs, antineoplastic agents, and strong acidic and alkaline drugs are at high risk of more severe drug extravasation events. Moreover, older nurses, first identified by nurses, and the infusion site close to the joint are nurse-related factors that affected the severity of drug extravasation. In order to prevent the occurrence of drug extravasation and reduce the severity of drug extravasation, the nurses should strengthen the learning of emergency plans related drug extravasation, strengthen 
inspections of high-risk patients. Besides, the managers should strengthen the risk warning management of high-risk extravasation drugs.

\section{Acknowledgments}

We would like to thank all the nursing managers and nurse leaders at Xiangya Hospital for their support and assistance in the process of collecting drug extravasation events.

\section{Funding}

This study was funded by National Natural Science Foundation of China (Grant No: 72174210). It was also supported by 2021 Xiangya Nursing Discipline Highlevel Scientific Research Project Cultivation Plan (Grant No: 2021xyhlpyjh004) and Project of Hunan Provincial Health Committee (Grant No: 202114050134).

\section{Disclosure}

The authors report no conflicts of interest in this work.

\section{References}

1. Le A, Patel S. Extravasation of noncytotoxic drugs: a review of the literature. Ann Pharmacother. 2014;48(7):870-886. doi:10.1177/ 1060028014527820

2. Gorski LA, Hadaway L, Hagle ME, et al. Infusion therapy standards of practice, 8th edition. J Infus Nurs. 2021;44(1S Suppl 1):S1-S224. doi:10.1097/NAN.0000000000000396

3. Goutos I, Cogswell LK, Giele H. Extravasation injuries: a review. $J$ Hand Surg Eur Vol. 2014;39(8):808-818. doi:10.1177/ 1753193413511921

4. Chiu K, Tindholdt TT, Tønseth KA. Extravasation injuries. Tidsskr nor Laegeforen. 2016;136(3):233-235. doi:10.4045/tidsskr.15.0230

5. Pluschnig U, Haslik W, Bartsch R, et al. Extravasation emergencies: state-of-the-art management and progress in clinical research. Memo. 2016;9(4):226-230. doi:10.1007/s12254-016-0304-2

6. Ching DL, Wong KY, Milroy C. Paediatric extravasation injuries: a review of 69 consecutive patients. Int $J$ Surg. 2014;12 (10):1036-1037. doi:10.1016/j.ijsu.2014.08.355

7. Shaqdan K, Aran S, Thrall J, et al. Incidence of contrast medium extravasation for CT and MRI in a large academic medical centre: a report on 502,391 injections. Clin Radiol. 2014;69(12):1264-1272. doi:10.1016/j.crad.2014.08.004

8. Thyoka M. Clinical negligence claims in pediatric surgery in England: pattern and trends. Eur J Pediatr Surg. 2015;25(1):66-70. doi:10.1055/s-0034-1395485
9. Kim JT, Park JY, Lee HJ, et al. Guidelines for the management of extravasation. J Educ Eval Health Prof. 2020;17(21):21. doi:10.3352/ jeehp.2020.17.21

10. Reynolds PM, MacLaren R, Mueller SW, et al. Management of extravasation injuries: a focused evaluation of noncytotoxic medications. Pharmacotherapy. 2014;34(6):617-632. doi:10.1002/ phar. 1396

11. Corbett M, Marshall D, Harden M, et al. Treatment of extravasation injuries in infants and young children: a scoping review and survey. Health Technol Assess (Rockv). 2018;22(46):1-112. doi:10.3310/ hta 22620

12. Boulanger J, Ducharme A, Dufour A, et al. Management of the extravasation of anti-neoplastic agents. Support Care Cancer. 2015;23(5):1459-1471. doi:10.1007/s00520-015-2635-7

13. Kreidieh FY, Moukadem HA, El Saghir NS. Overview, prevention and management of chemotherapy extravasation. World J Clin Oncol. 2016;7(1):87-97. doi:10.5306/wjco.v7.i1.87

14. Atay S, Sen S, Cukurlu D. Incidence of infiltration/extravasation in newborns using peripheral venous catheter and affecting factors. Rev Esc Enferm USP. 2018;52(e03360). doi:10.1590/s1980-220x2017 040103360

15. Frrat C, Erbatur S, Aytekin AH. Management of extravasation injuries: a retrospective study. J Plast Surg Hand Surg. 2013;47 (1):60-65. doi:10.3109/2000656X.2012.741065

16. Yan YM, Gong M, Chen JL, et al. Incidence, risk factors and treatment outcomes of drug extravasation in pediatric patients in China. Turk J Pediatr. 2017;59(2):1621. doi:10.24953/turkjped.2017.02.008

17. Yang Z, Huang L. Peripherally Inserted Central Venous Catheters [M]. Beijing: People's Medical Publishing House; 2021.

18. Hwang EJ, Shin C-I, Choi YH, et al. Frequency, outcome, and risk factors of contrast media extravasation in 142,651 intravenous contrast-enhanced CT scans. Eur Radiol. 2018;28(12):5368-5375. doi:10.1007/s00330-018-5507-y

19. Behzadi AH, Farooq Z, Newhouse JH, et al. MRI and CT contrast media extravasation: a systematic review. Medicine. 2018;97(9): e0055. doi:10.1097/MD.0000000000010055

20. Ozcan A, Baratalı E, Meral O, et al. Bullous dermatitis and skin necrosis developing after adrenalin extravasation. Eurasian $J$ Med. 2015;47(3):226-228. doi:10.5152/eurasianjmed.2015.58

21. Zhang Z, Ao L, He G, et al. Clinical value of high pressure resistant PICC catheter in MSCT examination of patients with nephrotic syndrome. Chin J CT MRI. 2019;17(09):104-106+52.

22. Loubani OM, Green RS. A systematic review of extravasation and local tissue injury from administration of vasopressors through peripheral intravenous catheters and central venous catheters. $J$ Crit Care. 2015;30(3):653.e9-17. doi:10.1016/j.jcrc.2015.01.014

23. Azaïs $\mathrm{H}$, Bresson L, Bassil A, et al. Chemotherapy drug extravasation in totally implantable venous access port systems: how effective is early surgical lavage? J Vasc Access. 2015;16(1):31-37. doi:10.5301/ jva.5000316
Risk Management and Healthcare Policy

\section{Publish your work in this journal}

Risk Management and Healthcare Policy is an international, peerreviewed, open access journal focusing on all aspects of public health, policy, and preventative measures to promote good health and improve morbidity and mortality in the population. The journal welcomes submitted papers covering original research, basic science, clinical \& epidemiological studies, reviews and evaluations, guidelines, expert opinion and commentary, case reports and extended reports. The manuscript management system is completely online and includes a very quick and fair peer-review system, which is all easy to use. Visit http://www.dovepress.com/testimonials.php to read real quotes from published authors. 